$\sqrt{18}$

TRANS * núm. $20 \cdot 2016$

ARTICULO · 57-74
Este trabajo nace en el ámbito de la Traducción e Interpretación (TI) y se enmarca en el área de formación de traductores en España. El estudio que presentamos forma parte de un proyecto más amplio de tesis doctoral cuyo objetivo general es promover la adquisición de la competencia intercultural (CI) del estudiantado de Traducción. Este artículo recoge una investigación de corte empírico a la vez que descriptivo, donde reunimos las percepciones de traductores profesionales sobre el papel que desempeña el factor cultural y concretamente, la $\mathrm{Cl}$, en su trabajo diario. Para ello utilizamos métodos cuantitativos (cuestionarios) y cualitativos (entrevistas) aunque el enfoque principal del estudio es cualitativo. El propósito de este trabajo es describir las implicaciones de la cultura en el mundo laboral de la traducción para introducir mejoras en la etapa de formación y dar un paso más hacia la reducción de la brecha existente entre formación y profesión (Kelly, 2005; Katan, 2009b; Pym et al. 2012).

PALABRAS CLAVE: Traducción, formación de traductores, competencia intercultural $(\mathrm{Cl})$, traductor profesional.

\title{
La competencia intercultural en la profesión del traductor: aproximación desde la formación de traductores y presentación de un estudio de caso
}

Silvia GutiérRez Bregón Universidad de Granada
The intercultural competence in the translator's profession: A translator training approach and the presentation of a case study

This piece of work originates in the field of Translation and Interpreting (TI) studies and it is framed within the area of translator training in Spain. The findings we present here constitute a specific part of our PhD research project, whose general objective is to promote the intercultural competence acquisition of translation students. This paper gathers empiric and descriptive data about professional translators' perceptions on the role of the cultural element and the IC in their daily work. Quantitave and qualitative methods (questionnaires and interviews) were employed although the general focus of the study is mainly qualitative. The aim of the research is to describe the actual implications of culture in the translator's job so improvements in translator training may be introduced and the gap between academic training and profession may be reduced (Kelly, 2005; Katan, 2009b; Pym et al. 2012).

KEY WORDS: Translation, translator training, intercultural competence (IC), professional translator. 


\section{ANTECEDENTES Y CONTEXTO}

En este apartado explicaremos cómo se ha ido forjando nuestro estudio en el marco de tres líneas principales de investigación: el EEES y la formación mediante competencias, la CI en formación de traductores, y la figura del traductor profesional en el mercado actual de la traducción.

\section{I.I. El EEES y la formación por competencias en $T I$}

Cuando comenzamos nuestra investigación doctoral sobre la adquisición de la CI de los estudiantes de traducción (Gutiérrez Bregón, 2009), la educación superior europea se encontraba inmersa en un importante cambio que supuso la implantación del Plan Bolonia (1999) y la formación de un espacio europeo común que regulase la educación superior: el EEES. En la actualidad los planes universitarios ya han sido adaptados a este nuevo contexto cuyo pilar fundamental en cuanto al paradigma metodológico educativo adoptado es la enseñanza mediante competencias y resultados de aprendizaje.

Si bien no es nuestra intención profundizar en cuestiones de diseño curricular, sí es relevante destacar que en el ámbito de la formación de traductores el enfoque pedagógico general que se seguía ya se situaba en sintonía con los objetivos del EEES en el momento del cambio (Kelly, 2005; Hurtado, 2007; Calvo et al., 2012). La línea de investigación abierta en formación de traductores sobre el concepto de competencia traductora y los métodos para la adquisición de ésta es anterior al EEEs (García Izquierdo, 20II). Por otra parte, otro de los objetivos de este nuevo marco educativo es acercar la formación a las necesidades del mercado laboral. En este sentido, la didáctica de la traducción actual se ha preocupado por acercar a los estudiantes la realidad de la profesión y a menudo se trasladan al aula encargos de traducción similares a los que se encontrará el alumno al finalizar sus estudios (Calvo et al., 20I2: 88). El presente trabajo implica un paso más hacia ese acercamiento entre formación y profesión, ya que profundiza en la influencia que tendría la CI en el trabajo diario del traductor para estudiar en una investigación posterior cómo trasladar los resultados obtenidos a la clase de traducción y conseguir que los estudiantes mejoren la adquisición de esta competencia.

\subsection{La CI en formación de traductores}

Este trabajo se inscribe en el ámbito de estudio del proyecto ACCI (Adquisición de la competencia cultural e intercultural en la formación de traductores), desarrollado por el grupo de investigación AVANTI (HUM-763) de la Universidad de Granada y financiado por el Plan Nacional I+D. Se trata de uno de los pocos proyectos que estudian la CI en formación de traductores, siendo la presencia de dicha competencia bastante limitada en la literatura de esta disciplina (Schäffner, 2003; Witte, 2008; Katan, 2009a; Gutiérrez Bregón, 2009; Gregorio, 2012; Yarosh y Muies, 20I2; PICT, 20I3). Asimismo, también son escasas las propuestas de modelos de competencia traductora donde estaría incluida la CI (Kelly, 2002; PACTE, 2008). Por lo tanto, tuvimos que acudir a ámbitos como los Estudios de Comunicación Intercultural para encontrar un corpus más amplio de definiciones, modelos y descripciones de CI que completasen la base teórica de nuestra investigación. En seguida descubrimos que sería muy enriquecedor que tanto los estudios de comunicación intercultural como los estudios de traducción trabajasen en paralelo. Robinson (2003: I94) ya señalaba la falta de colaboración de ambas disciplinas. 
Según este autor, los programas de comunicación intercultural estarían destinados a formar personas competentes en su propia cultura y en otras culturas, es decir, «mediadores culturales» que sepan desenvolverse tanto a nivel lingüístico como social en situaciones de interculturalidad. Podríamos pensar que la traducción estaría incluida aquí, sin embargo los expertos en comunicación intercultural dan por hecho que los traductores e intérpretes saben cómo actuar de forma eficaz en ámbitos multiculturales: «The ultimate goal of ICC [intercultural communication] is the base line of translator/ interpreter training» (Robinson, 2003: 95). En un intento por tender puentes y sentar las bases para un posible modelo de CI aplicable en TI, elaboramos una clasificación cronológica de los modelos de CI realizados hasta la fecha en el marco de los estudios de comunicación intercultural (Gregorio y Gutiérrez, en prensa) siendo conscientes de que se hacía cada vez más patente la necesidad de contar con una definición y un modelo específicos de CI adaptados a la formación de traductores, porque si bien los modelos disponibles en los estudios de comunicación intercultural constituyen una referencia importante, existe una diferencia fundamental entre una y otra disciplina en cuanto al concepto de comunicación que imposibilitaría una definición común y aplicable del concepto de CI. En los estudios de comunicación intercultural se trabaja con un tipo de comunicación directa y espontánea dada entre miembros de culturas distintas. El caso del traductor es diferente en este sentido porque el tipo de comunicación con la que trabaja es de carácter indirecto y artificial, ya que ocurre a través de textos. Además, el traductor no está presente cuando su texto es leído y, por tanto, no puede recibir feedback sobre su actuación. Se trata de una figura intermedia que facilita la interacción y el entendimiento a través de unas habilidades y conocimientos adquiridos para el desarrollo de su profesión, que son de naturaleza muy concreta (Schäffner, 2003).

Para el estudio que presentamos acudimos de 59 nuevo a los Estudios de Traducción y tomamos como referencia el modelo de competencia traductora (CT) de Kelly (2002) donde incluye y describe la competencia cultural ${ }^{\mathrm{T}}$. Necesitábamos un constructo teórico que también resultase operativo en un contexto empírico como el nuestro, por lo que el modelo de CT de esta autora con su correspondiente descripción de la CI desarrollado en el ámbito de la formación de traductores nos pareció el más apropiado. Además de estar adaptado a la enseñanzaaprendizaje de la traducción fue diseñado conforme a unos objetivos de formación concretos para cada subcompetencia que lo forma. Según la autora, la competencia cultural «comprende no sólo conocimientos enciclopédicos con respecto a los países donde se hablan las lenguas correspondientes, sino también sobre los valores, mitos, percepciones, creencias y comportamientos y sus representaciones textuales» (Kelly, 2002: I4). Y por tanto, los objetivos que según ella deberían guiar la práctica docente de la traducción en lo referente a la adquisición de esta competencia por parte de los estudiantes son los siguientes:

- Dar a conocer las principales instituciones de las culturas de las lenguas A, B y C, en su contexto histórico y social.

I Aunque existe un intenso debate sobre la denominación más apropiada para la CI a través de un baile intenso de nombres en la literatura correspondiente (sensibilidad cultural, competencia comunicativa intercultural, competencia cultural, etc.) nosotros tomamos el término intercultural en lugar de cultural porque subraya el carácter dinámico y la relación entre culturas del concepto. Si bien Kelly (2002) la denomina competencia cultural, esto no quiere decir que su concepción sea estática, al contrario, como se desprende de los componentes que la forman, según la autora. 
- Dar a conocer los valores, mitos, percepciones y creencias compartidos que condicionan los comportamientos de las diversas culturas de las lenguas A, B y C.

- Hacer comprender las relaciones existentes entre las culturas y sus textos.

- Además de utilizar este modelo de CT como referente teórico, lo incorporamos a las herramientas de recogida de información diseñadas para obtener la perspectiva del traductor profesional sobre la CI en su trabajo. Tanto en los cuestionarios como en las entrevistas se formulan cuestiones sobre las competencias descritas en el mismo.

\section{I.3. El traductor profesional}

Resulta obvio que el objetivo primordial de la formación de traductores es que los estudiantes adquieran un nivel de competencia traductora que le permita salir al mercado laboral suficientemente preparado como para cumplir las necesidades demandadas a su profesión, y que por tanto, debería existir un continuo entre la etapa de formación y la etapa profesional (Kelly, 2002, 2005; Calvo et al. 2012). Lo que no está tan claro es qué entendemos por traductor profesional o experto: ¿aquel que posee una titulación universitaria en TI? ¿Aquel que no tiene formación académica pero ha ejercido de traductor toda su vida? ¿Alguien que no ha terminado sus estudios pero ya cuenta con un contrato laboral para ejercer de traductor? Todas estas cuestiones nos surgieron a la hora de intentar definir un perfil concreto para elegir la muestra poblacional de nuestro estudio empírico. Según Kelly (2002: I6) un traductor experto es aquel que desarrolla actuaciones de éxito cumpliendo unos criterios de calidad (como puede ser satisfacer las necesidades de un cliente con un tiempo y un precio acordados).

Pym et al. (2012) abordan el asunto del estatus del traductor profesional de forma extensa.
$\mathrm{Su}$ trabajo y el desarrollado por Katan (2009b) recogen los resultados de encuestas realizadas a traductores de varios países de la Unión Europea, Estados Unidos, Canadá y Australia. Creemos que arrojan suficiente luz como para hacernos una idea del contexto actual en el que se encuentra la profesión del traductor y que resumimos en los puntos siguientes:

- Existe una falta de regulación en la profesión del traductor que acarrea consecuencias negativas como intrusismo laboral, bajos salarios, códigos de conducta y calidad inexistentes, y bajo nivel de asociacionismo.

- Existe falta de consenso entre los traductores en cuanto a la percepción de su profesión.

- El marcador de estatus más importante es la percepción de los clientes/empresas empleadoras.

- Según los modelos de profesionalización revisados desde la sociología, a la traducción le queda un largo camino por recorrer, encontrándose en los primeros estadios de dicho proceso.

- Según los propios traductores, las competencias, habilidades y pericia se obtienen a través de la experiencia laboral y no tanto en la etapa de formación académica (si la hubiese).

- Debido a la gran fragmentación de la profesión la mayoría de traductores son autónomos o freelance.

- También existe cierta fragmentación en las asociaciones profesionales españolas de traductores.

Katan (2009b: 150) pone en manos de los académicos de los Estudios de Traducción y de los docentes la tarea de "profesionalizar» la actividad de la traducción, para, de este modo, elaborar e inculcar unos principios que se correspondan con la realidad de la profesión, ya que según él, existe una brecha enorme entre la teoría y la práctica. Considera que si en la etapa 
de formación el traductor asume los principios y la definición de su rol, adquiriendo así una identidad como profesional, será más fácil alcanzar un nivel de profesionalización reconocido y una relación más productiva con el cliente, y en general, con el mercado laboral. Compartimos la visión de este autor y es por ello que planteamos el presente trabajo como una pequeña aportación a esa iniciativa de reducir esa brecha entre la teoría y la práctica de la profesión del traductor.

\section{PREGUNTAS DE INVESTIGACIÓN Y OBJETIVOS}

A continuación presentamos una tabla que recoge los objetivos que intentamos alcanzar en esta parte del estudio empírico y las preguntas de investigación correspondientes que se plantearon al inicio. También incluimos las dimensiones y las variables que manejamos para la elaboración del cuestionario y entrevistas posteriores. Cabe aclarar en este punto que las preguntas de investigación de la tabla no se trasladaron directamente al cuestionario y a las entrevistas puesto que se adaptó y modificó su formulación de acuerdo a las variables y a cada una de las herramientas. Por motivos de espacio nos hemos centrado únicamente en tres de las cinco dimensiones que comprenden la totalidad del trabajo.

\section{METODOLOGÍA}

Nuestro estudio se sitúa en las Ciencias Sociales, concretamente en el campo de la investigación educativa que tradicionalmente se ha caracterizado por albergar dos líneas metodológicas de trabajo: la cuantitativa y la cualitativa (Sandín-Esteban, 2003).
La mayoría de autores coinciden en afirmar que el ámbito de la investigación educativa ha estado dividido tradicionalmente en dos paradigmas sociológicos a menudo enfrentados: el positivista y el hermenéutico-interpretativo. Mediante la clasificación de paradigmas de Latorre et al. (1996: 44) situamos nuestra investigación en el paradigma interpretativo. Partimos de una perspectiva fenomenológica que nos lleva a estudiar la percepción del traductor profesional mediante métodos de corte cualitativo como la entrevista semiestructurada en profundidad (Taylor y Bogdan, 1987). También diseñamos y aplicamos un cuestionario a través del cual obtuvimos datos de carácter cuantitativo. Estos datos se analizaron con técnicas estadísticas, pero hay que tener en cuenta que las variables empíricas que manejamos eran independientes y cualitativas (Cea D'Ancona, I996:I28), lo que le añade carácter interpretativo al análisis. Con todo, se alejaría de la estadística inferencial que se caracteriza por la generalización de datos de la muestra al conjunto de la población y se quedaría en la estadística descriptiva (Hueso y Cascant, 20I2: 38). Creemos que queda suficientemente marcado el enfoque inductivo y descriptivo del estudio, que deja a un lado lo experimental al partir de unas preguntas de investigación concretas en vez de hipótesis y cuyo objetivo es describir e interpretar una realidad determinada en un momento concreto en lugar de intentar corroborar hipótesis prefijadas a través de planteamientos estadísticos.

\section{I. Cuestionarios}

Además de intentar cumplir parcialmente los objetivos planteados en la tabla anterior (el volumen de información obtenido se completaría y enriquecería con las entrevistas posteriores), el cuestionario fue diseñado para configurar un perfil de traductor profesional 
TABLA I.

PREGUNTAS DE INVESTIGACIÓN

VARIABLES EMPÍRICAS

\begin{tabular}{|c|c|c|c|}
\hline \multirow{5}{*}{$\begin{array}{l}\text { Obtener información } \\
\text { sobre el perfil profe- } \\
\text { sional del traductor } \\
\text { encuestado }\end{array}$} & \multirow{5}{*}{$\begin{array}{l}\text { (I) Perfil profe- } \\
\text { sional }\end{array}$} & \multirow{2}{*}{$\begin{array}{l}\text { ¿Qué perfil académico y profe- } \\
\text { sional tiene? }\end{array}$} & Formación académica \\
\hline & & & Vinculación laboral actual \\
\hline & & \multirow{3}{*}{$\begin{array}{l}\text { ¿Cuáles son sus lenguas de } \\
\text { trabajo habituales y el tipo de } \\
\text { traducciones que realiza? }\end{array}$} & Lenguas de trabajo \\
\hline & & & $\begin{array}{l}\text { Direccionalidad de las traduc- } \\
\text { ciones que realiza }\end{array}$ \\
\hline & & & $\begin{array}{l}\text { Rama de la traducción a la } \\
\text { que se dedica }\end{array}$ \\
\hline \multirow{4}{*}{$\begin{array}{l}\text { Conocer de qué } \\
\text { modo percibe el tra- } \\
\text { ductor profesional la } \\
\text { CI en su trabajo dia- } \\
\text { rio y su relación con } \\
\text { otras competencias } \\
\text { de la CT }\end{array}$} & \multirow{4}{*}{$\begin{array}{l}\text { (2) Percepción de } \\
\text { la CI en el marco } \\
\text { de la CT }\end{array}$} & $\begin{array}{l}¿ Q \text { Qué lugar ocupa la CI respecto } \\
\text { a otras competencias de la CT? }\end{array}$ & $\begin{array}{l}\text { Valoración de la cI en relación } \\
\text { con otras competencias del } \\
\text { traductor }\end{array}$ \\
\hline & & \multirow{2}{*}{$\begin{array}{l}\text { ¿Qué opinión tiene el traductor } \\
\text { sobre el papel de la CI en su } \\
\text { profesión? }\end{array}$} & $\begin{array}{l}\text { Valoración del conocimiento } \\
\text { sobre otras culturas }\end{array}$ \\
\hline & & & $\begin{array}{l}\text { Actitud hacia el aprendizaje } \\
\text { intercultural }\end{array}$ \\
\hline & & $\begin{array}{l}\text { ¿La relevancia de la CI depende } \\
\text { de la especialidad de traducción } \\
\text { a la que se dedica? }\end{array}$ & $\begin{array}{l}\text { Relación entre CI y diferentes } \\
\text { ramas de la traducción }\end{array}$ \\
\hline \multirow{2}{*}{$\begin{array}{l}\text { Averiguar de qué } \\
\text { forma concibe el tra- } \\
\text { ductor la CI y su pro- } \\
\text { ceso de adquisición }\end{array}$} & \multirow{2}{*}{$\begin{array}{l}\text { (3) Aproximación } \\
\text { al concepto de CI } \\
\text { y su adquisición }\end{array}$} & $\begin{array}{l}¿ \text { ¿ué conocimientos, actitudes y } \\
\text { habilidades sobre otras culturas } \\
\text { considera el traductor que son } \\
\text { necesarios en su profesión? }\end{array}$ & Concepto de CI \\
\hline & & $\begin{array}{l}\text { ¿Cómo entiende el traductor el } \\
\text { proceso de adquisición de la CI? }\end{array}$ & $\begin{array}{l}\text { Etapa de adquisición más } \\
\text { favorable }\end{array}$ \\
\hline
\end{tabular}

experimentado, con una visión amplia y a la vez precisa de su actividad, capaz de reflexionar sobre el papel que desempeña la cultura en el día a día de su trabajo, y así poder realizar una selección de traductores que estuvieran dispuestos a participar en las entrevistas y pudieran aportar un volumen mucho mayor y más profundo de información sobre este aspecto.

Dado el tamaño de la muestra, nuestra intención no era extrapolar resultados al resto de la comunidad de traductores profesionales, sino describir e interpretar una realidad concreta para comprenderla mejor. De este modo, se 
podrían extraer una serie de tendencias que servirían como indicadores para continuar investigando en una dirección concreta. Planteamos una herramienta de tipo electrónico cuyo diseño realizamos mediante el programa Adobe Acrobat (X Pro) y su aplicación de creación de formularios. Este cuestionario pasó posteriormente una prueba de pilotaje con cuatro sujetos cuyos perfiles elegimos en función de los criterios que utilizamos posteriormente para el resto de la muestra y que describimos en la página siguiente. Dichos sujetos no reportaron ningún problema en cuanto a la estructura y planteamiento de las preguntas. Éramos conscientes de que el porcentaje de respuestas a un cuestionario electrónico es generalmente muy bajo. En función del piloto calculamos un volumen de respuestas del $30-35 \%$. La captación de participantes la realizamos a través de la plataforma online ProZ donde pudimos seleccionar una variedad amplia de perfiles que se ajustaban a unos requisitos propuestos previamente a partir de las conclusiones que sacamos de los estudios de Pym et al. (2012) y Katan (2009b) sobre el estatus del traductor profesional. Estos criterios que configuraron el perfil de la muestra fueron los siguientes:

- Que los traductores desarrollasen su actividad en el mercado de la traducción español.

- Que el español fuese su lengua de instrucción y de trabajo.

- Que tuviesen un mínimo de cinco años de experiencia como traductores.

- Que hubiese diversidad de especialidades de traducción en el grupo de participantes.

Manejamos un listado de más de 190 traductores con los que se contactó por correo electrónico, de los cuales 70 devolvieron el cuestionario completado. Al final hubo que descartar 5 por inconsistencias en varias respuestas, por lo que la muestra final quedó en 65 cuestionarios. En cuanto a las preguntas, incluimos una tipología amplia: preguntas cerradas con varias opciones a elegir, preguntas formuladas en escala Likert de opinión y de frecuencia (Oppenheim, 200o) 63 y preguntas totalmente abiertas. Conscientes de que el análisis sería más complejo cuanta mayor fuese la diversidad en las preguntas, asumimos esta dificultad a sabiendas de que obtendríamos mayor información y más variada. Para la gestión y el análisis de los datos se utilizó el programa Microsoft Excel (2007).

\subsection{Entrevistas}

Las entrevistas que realizamos se clasifican como individuales, semiestructuradas y en profundidad (Taylor y Bogdan, I984: IOI). Al tratarse de entrevistas semiestructuradas, se elaboró un guión previo con los puntos más importantes sabiendo de antemano que la formulación de las preguntas variaría en cada entrevista. Creímos conveniente primar el factor espontaneidad porque de este modo los entrevistados se sentirían más cómodos en un ambiente distendido. Esto no se hubiera logrado si hubiésemos estado pendientes del guión en todo momento, por lo que hubo ocasiones en los que dejamos fluir la conversación sabiendo que estábamos alterando el guión fijado previamente.

Se eligieron ocho participantes de entre todos los que respondieron el cuestionario intentando cubrir la mayoría de especialidades de traducción posibles. Contactamos con ellos a través de correo electrónico y para la realización de las entrevistas nos desplazamos a diferentes lugares de España. La duración media de cada una de ellas osciló entre los 20 y los 40 minutos $y$ fueron registradas mediante una grabadora digital de voz. El objetivo fundamental era profundizar en las dimensiones planteadas al comienzo y que por cuestiones de formato, el cuestionario quedaba bastante limitado. No 
buscábamos en este caso lograr consenso en las respuestas sino abordar las preguntas de investigación desde todas las ramas de la traducción 64 posibles con candidatos capaces de aportar un volumen de información extenso y relevante. Una vez realizadas las entrevistas se procedió a transcribir, codificar y categorizar cada una de ellas (Soriano, 2007: 192) para facilitar su análisis posterior.

Finalmente cabe apuntar que hemos optado por una triangulación de métodos (Taylor y Bogdan, I984: 92; Ruiz-Olabuénaga, 20I2: Io9) combinando herramientas cuantitativas (cuestionarios) y cualitativas (entrevistas) para dotar de mayor validez y fiabilidad a nuestro estudio. Nuestra intención no era la de confrontar datos sino completar o ampliar la información recogida a partir de ambas técnicas.

\section{RESULTADOS}

A continuación presentamos los resultados obtenidos en función de los objetivos y preguntas de investigación planteados en la tabla I. Diferenciaremos entre cuestionario y entrevistas.

\section{I. Objetivo i: Obtener información sobre el perfil profesional del traductor encuestado} ¿Qué perfil académico y profesional tiene?

Cuestionario: La mayoría eran traductores autónomos a jornada completa con formación académica mayoritariamente en Traducción o Filología, aunque muchos tenían formación en áreas técnicas que habían complementado con un posgrado en Traducción. Casi el $70 \%$ contaba con una experiencia laboral de entre 5 y 15 años en el campo de la traducción. El $30 \%$ restante tenían más de I5 años de experiencia. Entrevistas: Al igual que para el cuestionario,

seleccionamos participantes con perfiles muy diversos que hemos resumido a continuación en la tabla 2.

¿Cuáles son sus lenguas de trabajo habituales $y$ el tipo de traducciones que realiza?

Cuestionario: La combinación de lenguas de trabajo mayoritaria es español e inglés, traduciendo casi siempre hacia el español. En cuanto a las ramas de especialización, contamos con perfiles muy heterogéneos en este sentido cubriendo así un campo amplio de especialidades, que ordenamos por frecuencia de respuestas: traducción económica, jurídica, comercial, científico-técnica, localización web y videojuegos, traducción audiovisual, traducciones de textos turísticos, publicitarios y de prensa, y traducción literaria.

Entrevistas: Cabe apuntar que algunos traductores abarcaban varias especialidades, como se observa en la siguiente tabla (tabla 2) donde resumimos el perfil de cada uno de ellos.

4.2. Objetivo II: Conocer de qué modo percibe el traductor profesional la CI en su trabajo diario y su relación con otras competencias de la CT

¿Qué lugar ocupa la CI respecto a otras competencias de la CT?

Cuestionario: Tomando como referencia el modelo de competencia traductora de Kelly (2002), planteamos una serie de definiciones de competencias que tenían que valorar como «muy importante, importante, poco importante o nada importante». Lo primero que nos llamó la atención fueron las dos competencias que más sobresalían en la valoración «muy importante»: la estratégica y la instrumental. Respecto a la CI, más de un $95 \%$ de los encuestados la valoraron como importante o muy importante seguida 
TABLA 2. PRESENTACIÓN DEL PERFIL PROFESIONAL DE LOS TRADUCTORES ENTREVISTADOS

\begin{tabular}{|c|c|c|c|}
\hline & $\begin{array}{l}\text { FORMACIÓN ACADÉMICA Y } \\
\text { VINCULACIÓN LABORAL }\end{array}$ & $\begin{array}{c}\text { LENGUAS DE TRABAJO Y } \\
\text { DIRECCIONALIDAD DE LAS } \\
\text { TRADUCCIONES }\end{array}$ & RAMA DE ESPECIALIDAD \\
\hline $\begin{array}{l}\text { SUJETO } \\
\text { I }\end{array}$ & $\begin{array}{l}\text { - Licenciado en TI } \\
\text { - Máster en estudios asiáticos } \\
\text { - Autónomo } \\
\text { - } 5 \text { años de experiencia }\end{array}$ & $\begin{array}{l}\text { - Inglés > Español } \\
\text { - Alemán >Español } \\
\text { - Chino >Español }\end{array}$ & $\begin{array}{l}\text { - Videojuegos } \\
\text { - Localización de software y } \\
\text { apps (móviles) } \\
\text { - Textos periodísticos } \\
\text { - Textos jurídicos y técnicos } \\
\text { (puntualmente) }\end{array}$ \\
\hline $\begin{array}{l}\text { SUJETO } \\
2\end{array}$ & $\begin{array}{l}\text { - Sin formación académica } \\
\text { - Programador informático } \\
\text { convertido en traductor } \\
\text { - Autónomo } \\
\text { - Más de } 20 \text { años de experiencia }\end{array}$ & $\begin{array}{l}\text { - Alemán >Español } \\
\text { - Inglés > Español }\end{array}$ & $\begin{array}{l}\text { - Textos técnicos } \\
\text { - Textos promocionales y de } \\
\text { marketing (puntualmente) }\end{array}$ \\
\hline $\begin{array}{l}\text { SUJETO } \\
3\end{array}$ & $\begin{array}{l}\text { - Licenciada en periodismo y TI } \\
\text { - Máster en radio } \\
\text { - Autónoma } \\
\text { - } 8 \text { años de experiencia }\end{array}$ & $\begin{array}{l}\text { - Inglés }>\text { Español } \\
\text { - Francés }>\text { Español } \\
\text { - Polaco }>\text { Español } \\
\text { - Italiano (puntual) >Español }\end{array}$ & $\begin{array}{l}\text { - Textos periodísticos } \\
\text { - Textos de marketing y publi- } \\
\text { cidad } \\
\text { - Textos literarios (puntualmente) } \\
\text { - Textos técnicos (puntualmente) }\end{array}$ \\
\hline $\begin{array}{c}\text { SUJETO } \\
4\end{array}$ & $\begin{array}{l}\text { - Lda. en Filología inglesa } \\
\text { - Licenciada en TI } \\
\text { - Actualmente formándose } \\
\text { como intérprete } \\
\text { - Autónoma } \\
\text { - Más de I5 años de experiencia }\end{array}$ & Inglés>Español>Inglés & $\begin{array}{l}\text { - Textos médicos, farmacéuticos } \\
\text { y técnicos }\end{array}$ \\
\hline $\begin{array}{l}\text { SUJETO } \\
\quad 5\end{array}$ & $\begin{array}{l}\text { - Licenciado en TI } \\
\text { - Máster en traducción audiovisual } \\
\text { - Freelance y traductor en una } \\
\text { multinacional tecnológica } \\
\text { - } 7 \text { años de experiencia }\end{array}$ & Inglés $>$ Español & $\begin{array}{l}\text { - Videojuegos } \\
\text { - Localización de software }\end{array}$ \\
\hline $\begin{array}{l}\text { SUJETO } \\
6\end{array}$ & $\begin{array}{l}\text { - Licenciada en TI } \\
\text { - Autónoma } \\
\text { - i2 años de experiencia }\end{array}$ & $\begin{array}{l}\text { Polaco }>\text { Español }>\text { Polaco } \\
\text { Inglés }>\text { Español y/o Polaco } \\
\text { Ruso }>\text { Español y/o Polaco } \\
\text { Catalán }>\text { Español y/o Polaco }\end{array}$ & $\begin{array}{l}\text { - Textos jurídicos } \\
\text { - Textos turísticos } \\
\text { - Textos económicos y empresariales } \\
\text { - Textos técnicos (puntualmente) }\end{array}$ \\
\hline $\begin{array}{l}\text { SUJETo } \\
7\end{array}$ & $\begin{array}{l}\text { - Licenciada en TI y Comu- } \\
\text { nicación Audiovisual } \\
\text { - Máster en TI (perfil investigador) } \\
\text { - Traductora para empresa } \\
\text { - } 8 \text { años de experiencia }\end{array}$ & $\begin{array}{l}\text { Inglés }>\text { Español } \\
\text { Francés }>\text { Español }\end{array}$ & $\begin{array}{l}\text { - Textos médicos, farmacéuticos } \\
\text { y técnicos }\end{array}$ \\
\hline $\begin{array}{l}\text { SUJETO } \\
8\end{array}$ & $\begin{array}{l}\text { - Licenciada en TI } \\
\text { - Autónoma } \\
\text { - Más de I5 años de expe- } \\
\text { riencia }\end{array}$ & Español>Inglés>Español & $\begin{array}{l}\text { - Textos técnicos } \\
\text { - Textos jurídicos } \\
\text { - Textos turísticos } \\
\text { - Textos periodísticos }\end{array}$ \\
\hline
\end{tabular}


de un $92 \%$ por la comunicativa y textual. La competencia peor valorada, con diferencia, fue la temática, calificada por un $40 \%$ como poco 66 importante y por un $15 \%$ como nada importante.

Entrevistas: Los participantes situaron la CI al mismo nivel que el resto de habilidades o competencias del traductor cuando se les preguntó si consideraban la CI como una habilidad complementaria de otras competencias como la lingüística/comunicativa. En todos estaba presente la idea de que no es posible aprender una lengua sin aprender paralelamente su cultura y añadieron que el contexto que rodea al texto es fundamental en una traducción. Algunos llegaron a afirmar que lengua es cultura. Nos pareció curioso que los traductores de la rama científico-técnica respaldasen también esta percepción, dado que en las respuestas a los cuestionarios se recogía la idea generalizada de que estos textos albergan escasa carga cultural o están desprovistos de ella, tal y como se observa en el apartado correspondiente (pregunta de investigación: ¿La relevancia de la CI depende de la especialidad de traducción a la que se dedica?)

S2: Deben estar al mismo nivel y además pienso que deben formar parte de la propia personalidad del traductor. El hecho de tener interés por las otras culturas, tener curiosidad, tener ganas de ir más allá de lo que te está pidiendo un texto en particular.

S8: Me parece FUndamental. Absolutamente. Es que NO puedo concebir la traducción sin la cultura. Es que (...) NO es concebible. Simplemente.

\section{¿Qué opinión tiene el traductor sobre el papel de la CI en su profesión?}

Cuestionario: Una gran mayoría de traductores concebían la traducción como una forma de comunicación intercultural, aunque casi un $45 \%$ consideraba que el conocimiento de otras cultu-

ras no tiene por qué ser decisivo para la buena o mala calidad de una traducción. Respecto a su actitud frente al aprendizaje intercultural, en general era muy positiva: consideraban que la cultura es un elemento presente en todo el proceso de traducción y es necesario esforzarse para seguir aprendiendo y no quedarse desactualizado, o bien mediante viajes, o siguiendo la actualidad de los países de las lenguas de trabajo a través del cine, la televisión o internet.

Entrevistas: Los ocho traductores entrevistados, teniendo en cuenta que cubrían especialidades de traducción muy distintas, coincidieron en que el conocimiento sobre otras culturas es una herramienta importantísima en su trabajo y reconocieron que se esforzaban por ampliar su aprendizaje intercultural día a día, mostrando una actitud proactiva y abierta. Nos pareció destacable que muchos mencionasen las ventajas de participar en programas de movilidad durante la etapa de formación.

$\mathrm{S}_{4}$ : Yo creo que es ESENCial [...] Te das cuenta de que las dificultades en realidad no están en las palabras técnicas y demás que te las aprendes. Haces glosarios y te las aprendes. El problema está en EL DÍA A DÍA, es decir, en cuestiones muy simples, en palabras muy simples que se utilizan. Pues las frases hechas de cada sitio. Todas esas cosas las tienes que conocer. Entonces incluso en traducción, yo también hago revisión, y me he encontrado con traducciones en las que el traductor no ha entendido la frase porque no tenía el concepto cultural, entonces una frase que a lo mejor el técnico que la escribió la hizo muy localizada, no ha sabido interpretar eso [refiriéndose al traductor].

$\mathrm{S}_{5}$ : Pues yo creo que idealmente es necesario vivir aunque sean nueve meses con alguna beca Erasmus durante tu formación o en tu trabajo más adelante... VIVIR en el país extranjero. Yo creo que incluso aunque que vivas en otro país 
[refiriéndose a países distintos a sus lenguas de trabajo], yo qué sé, Noruega o así, te va a ayudar a darte cuenta de que la cultura en este caso noruega, es diferente a la española, y eso como que te va a abrir un poco la mente.

\section{${ }_{\dot{L}}$ La relevancia de la CI depende de la especialidad de traducción a la que se dedica?}

Cuestionario: A pesar de considerar que la cultura es una constante en su trabajo, casi el 90 $\%$ reconocieron que el nivel de $\mathrm{CI}$ de un traductor estaría supeditado al tipo de traducciones que realiza. Muchos matizaron la respuesta, pero el pensamiento general recogido a través de esta herramienta es que en los textos científicos o técnicos la presencia de la cultura sería mínima y por tanto, también lo sería la relevancia de esta competencia. Sin embargo, en un texto literario la CI sería de máxima importancia. La visión de los traductores técnicos en este sentido nos pareció fundamental, por eso entrevistamos a varios profesionales de esta especialidad con el fin de ampliar dicha cuestión.

Entrevistas: La mayoría de los entrevistados coincidieron con la respuesta mayoritaria de los cuestionarios en que generalmente se necesita de más habilidad o conocimiento intercultural para traducir según qué textos (se mencionan literarios, turísticos, publicitarios y jurídicos mayoritariamente) en comparación con otros que no albergarían tanto contenido cultural (se mencionan los textos científicos y técnicos). Sin embargo, los traductores técnicos y científicos comentaban que esto no significaría que la totalidad de los textos técnicos estuvieran desprovistos completamente de carga cultural: la cultura aquí se suele manifestar, según ellos, de forma más velada o transversal, y por eso es necesario tener un conocimiento muy profundo de la cultura de origen del texto. Coincidieron en que los textos que trabajan sin contenido cultural alguno, como los manuales, son minoritarios, así como también lo es el perfil de traductor técnico que traduce estos textos de forma exclusiva.

Apreciamos un sesgo importante en este punto respecto a la concepción de CI que albergaban los traductores encuestados. Su idea de CI era bastante superficial, dado que la entendían únicamente desde una perspectiva micro textual al constatar que el nivel de CI requerido dependería del tipo de traducciones que se realicen, sin tener en cuenta dimensiones de mayor profundidad que abarcaría esta competencia (comportamientos, valores, etc). Por lo tanto, partirían de una concepción de CI estática. Además, la mayoría de ellos afirmaba mantener el contacto con otras culturas a través de la prensa, la literatura, el cine, y mediante viajes puntuales a otros países. Estas actividades conducirían a la adquisición de conocimientos de tipo más declarativo que experiencial, y dejarían a un lado iniciativas que fomentasen la dimensión interpersonal y de interacción de la CI (Byram, 1997).

S2: Bueno, no sé, puede haber traductores técnicos que se dediquen el $90 \%$ de su tiempo a traducir por ejemplo manuales, pues claro, obviamente si traduces manuales te vas a encontrar con menos carga cultural. Pero si te ocurre como en mi caso que traduces TODO lo que un cliente necesita, no solo los manuales sino también muchos materiales más, enseguida te vas a encontrar cosas que tienes que conocer. $Y$ en este sentido no es solo tener un glosario en la cabeza de que esta palabra se traduce de esta otra manera, sino es comprender por qué tengo que utilizar esta palabra.

S7: Yo hago traducciones científicas y técnicas, y aunque en principio parece que tiene que ser un lenguaje desprovisto de referencias culturales, en realidad te las encuentras de forma 
transversal. Entonces son cosas que tienes que ir sorteando.

68

4.3. Objetivo III: Averiguar de qué forma concibe el traductor la CI y su proceso de adquisición

¿Qué conocimientos, actitudes y babilidades sobre otras culturas considera el traductor que son necesarios en su profesión?

Cuestionario: En cuanto a la percepción del concepto de CI, fue complicado formular una pregunta de cuestionario que no contuviese demasiada información para no limitar las respuestas evitando ser muy intrusivos, y al mismo tiempo que no quedase totalmente abierta para que las respuestas se ajustasen a nuestra pregunta de investigación. Intentamos situarnos en un punto intermedio: formulamos una pregunta abierta pero en cuyo enunciado se les pedía que enumerasen habilidades, actitudes y conocimientos, que son las dimensiones que enmarcan algunos modelos de CI de referencia (Byram, 1997 y 2013; Deardorff, 2006 y 2009). En la tabla 3 mostramos los elementos que más veces se mencionaron:

Nos llama la atención las numerosas coincidencias que encontramos entre los elementos de la tabla señalados por los traductores con el listado de conocimientos, habilidades y actitudes que configuran el modelo de Byram (1997), que si bien está orientado hacia la enseñanza de lenguas extranjeras, estas similitudes lo podrían hacer extensible también a la formación de

TABLA 3

APRoXimACión AL CONCEPTO DE CI (CUESTIONARIo)

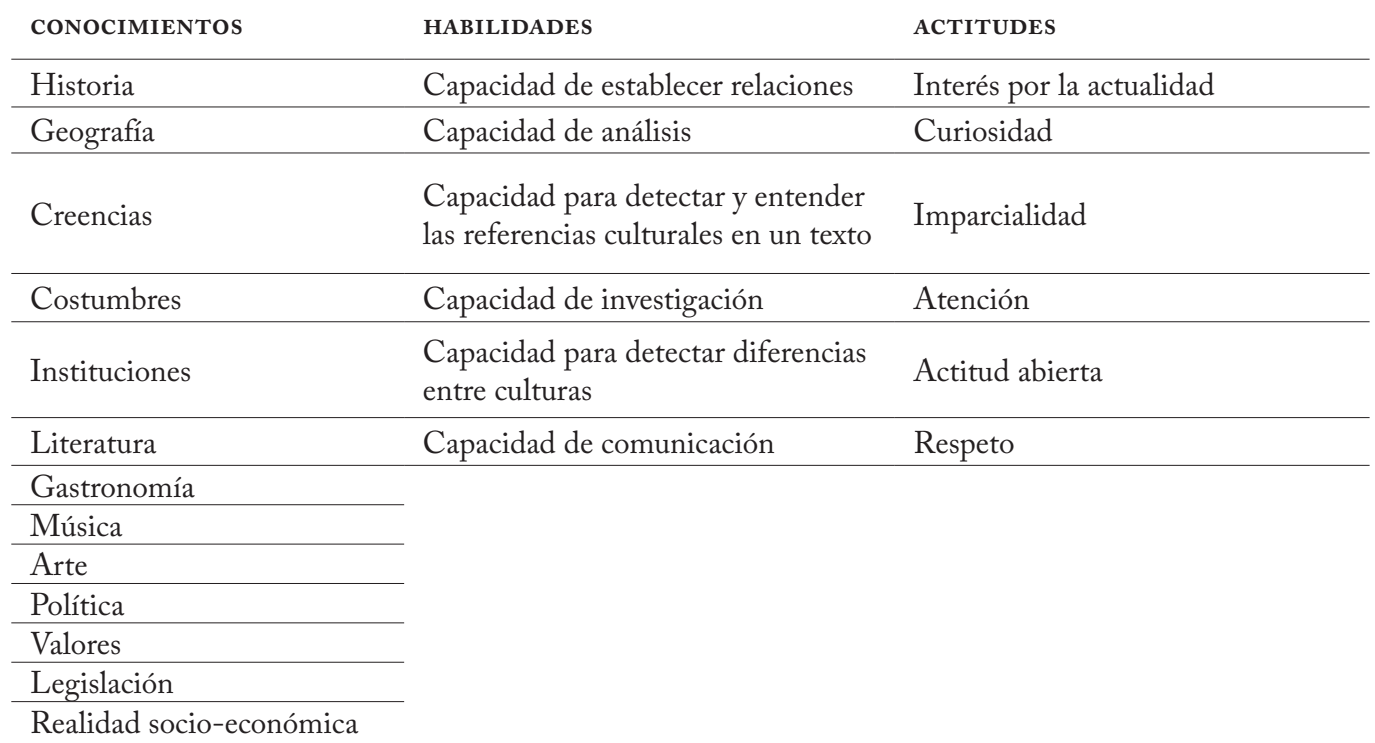


traductores. No obstante, insistimos en que la mayoría de los factores mencionados por los traductores profesionales se ciñen a una idea de
CI orientada a la dimensión textual sin incidir tanto en aspectos interpersonales y de interacción.

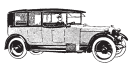

69

TABLA 4 .

APROXIMACIÓN AL CONCEPTO DE CI (ENTREVISTAS)

\begin{tabular}{|c|c|c|c|}
\hline SUJETOS & CONOCIMIENTOS & HABILIDADES & ACTITUDES \\
\hline $\mathrm{S}_{\mathrm{I}}$ & $\begin{array}{l}\text { Historia, cultura pop, reli- } \\
\text { gión, literatura, geografía. }\end{array}$ & & $\begin{array}{l}\text { Estar libre de prejuicios, } \\
\text { curiosidad, proactividad. }\end{array}$ \\
\hline $\mathrm{S}_{2}$ & $\begin{array}{l}\text { Historia, arte, literatura, } \\
\text { modismos y juegos de pala- } \\
\text { bras. }\end{array}$ & $\begin{array}{l}\text { Interpersonales y comunica- } \\
\text { tivas para no cometer errores } \\
\text { de tipo cultural con el cliente; } \\
\text { saber interiorizar la informa- } \\
\text { ción para poder profundizar } \\
\text { en el análisis de un texto. }\end{array}$ & $\begin{array}{l}\text { Estar abierto a otros puntos } \\
\text { de vista, empatía, curio- } \\
\text { sidad, esfuerzo e incon- } \\
\text { formismo con la primera } \\
\text { opción de traducción. }\end{array}$ \\
\hline $\mathrm{S}_{3}$ & $\begin{array}{l}\text { Contexto socio-cultural de } \\
\text { las lenguas de trabajo, histo- } \\
\text { ria, literatura, costumbres. }\end{array}$ & $\begin{array}{l}\text { Disciplina para desarrollar } \\
\text { una formación intercultural } \\
\text { continua. }\end{array}$ & Ser abierto y curioso. \\
\hline $\mathrm{S}_{4}$ & $\begin{array}{l}\text { Los conocimientos sobre } \\
\text { otras culturas que se apren- } \\
\text { den durante la formación. }\end{array}$ & $\begin{array}{l}\text { Ser capaz de entender el } \\
\text { contexto en profundidad y } \\
\text { saber transmitir el mensaje } \\
\text { correctamente. Capacidad de } \\
\text { investigación. }\end{array}$ & $\begin{array}{l}\text { Interés por la lectura y } \\
\text { curiosidad ya que es la llave } \\
\text { para el resto. (motivación y } \\
\text { esfuerzo). }\end{array}$ \\
\hline $\mathrm{S}_{5}$ & $\begin{array}{l}\text { Conocimientos sobre las cul- } \\
\text { turas del mundo en general. }\end{array}$ & $\begin{array}{l}\text { Capacidad para saber docu- } \\
\text { mentarse acerca de temas } \\
\text { culturales. }\end{array}$ & $\begin{array}{l}\text { Tener iniciativa, estar atento } \\
\text { y ser curioso. }\end{array}$ \\
\hline S6 & & & $\begin{array}{l}\text { Ser muy curioso y abierto, } \\
\text { tener mucho interés por } \\
\text { aprender de nuevas culturas } \\
\text { y buena voluntad por hacer } \\
\text { esfuerzos en cuanto a tiem- } \\
\text { po y dinero para viajar. }\end{array}$ \\
\hline
\end{tabular}

Historia, geografía, usos y

S7 costumbres y conocimientos

sobre la actualidad social de

Capacidad para cuestionar puntos de vista propios.
Curiosidad. las lenguas de trabajo. 
Entrevistas: En la tabla 4 presentamos las respuestas resumidas a la pregunta sobre la percepción del concepto de CI, donde se les instó, al igual que en el cuestionario, a que mencionasen componentes de cada una de las dimensiones que formarían la CI (conocimientos, habilidades y actitudes). Algunos participantes no aludieron a todas las dimensiones, sino que se limitaron a responder de forma más genérica, por eso hay algún espacio en blanco en la tabla. Concretamente los sujetos 6 y 8 situaron el factor «actitudes» como el más relevante y del que dependería el resto, por lo que se centraron en describir solamente esta dimensión.

Destacamos al igual que en la tabla realizada para el cuestionario, las coincidencias con el modelo de CI de Byram (1997). Llama la atención que en el apartado de actitudes todos los entrevistados hagan mención a la curiosidad como un factor fundamental en el desarrollo de la CI del traductor profesional.

\section{¿Cómo entiende el traductor el proceso de adquisición de la CI?}

Cuestionario: Los traductores que respondieron el cuestionario consideran que la mejor etapa para empezar a aprender sobre otras culturas sería durante su formación universitaria, que este aprendizaje es un proceso continuado que se extendería también a la etapa profesional y que esta última es clave para su adquisición, dado que en su trabajo se enfrentan a una experiencia directa y a conflictos reales. Optaron por que durante la formación, el factor cultural se distribuyese a lo largo de todo el plan de estudios, de manera que estuviese presente de forma homogénea en todas las asignaturas y no tanto en materias aisladas.

Entrevistas: Respecto al periodo y el proceso de adquisición, nos pareció muy importante que seis de los ocho entrevistados respondiesen que lo más apropiado sería la implementación de una pedagogía intercultural antes incluso de la formación universitaria. De entre ellos, algunos consideraban que lo mejor es comenzar desde la infancia a inculcar este tipo de competencias. Los otros dos traductores se decantaron por que el comienzo de este aprendizaje fuese durante la etapa de formación universitaria, en la línea de las respuestas recogidas en el cuestionario, ya que según su propia experiencia, es cuando más tiempo libre habría para reforzarlo con viajes y estancias en el extranjero. Por otra parte, los profesionales consideraron imprescindible un enfoque transversal a la hora de redactar del plan de estudios, y también aludieron a la responsabilidad del profesor de traducción en este sentido, al ser el encargado de elegir los textos. La mayoría de ellos también ven muy útiles las asignaturas específicas de cultura, como Civilización, aunque alguno señaló que a menudo se les da un enfoque demasiado teórico.

Sı: Cuanto antes. Incluso antes de plantearte que quieres ser un traductor, tienes que tener una curiosidad y una base porque te va a ayudar mucho. Yo lo noté mucho en mi periodo de formación que había gente bilingüe o gente que había tenido contacto con otros países desde muy pequeña y que nos llevaba ventaja.

S6: Yo creo que esas asignaturas [refiriéndose a las específicas de cultura] Sí son buenas de por sí. Y luego hay mucho conocimiento que se adquiere a través de los textos que se traducen en clase. Con que realmente, creo que con una BUENA selección de los textos con los que se trabaja, que son de todos modos en muchísimas ocasiones, competencia del profesor, con una buena orientación de este lado, sí se pueden conseguir también los mismos objetivos. 


\section{CONCLUSIONES Y VALORACIÓN GLOBAL}

A partir de los resultados obtenidos mediante las dos herramientas llegamos a unas conclusiones que presentamos en función de las dimensiones establecidas con anterioridad (Tabla I). Posteriormente realizamos una valoración general de lo que implicarían dichos resultados para la formación de traductores en España.

\section{Perfil profesional}

- La mayoría de traductores encuestados cuentan con el título de TI.

- Tienen un perfil polivalente en cuanto a especialidades de traducción, abarcando muchos de ellos varias ramas, aunque observamos una tendencia a especializarse en los tipos de traducción más demandados por el mercado, como la económica y jurídica, la científico-técnica o la localización de software.

\section{PERCEPCIÓN DE LA CI EN EL MARCO DE LA CT}

- Los participantes en los cuestionarios reconocieron la relevancia de la cultura en su trabajo y situaron la CI en tercer lugar de importancia respecto al resto de competencias, por debajo de la estratégica y de la instrumental. Los traductores entrevistados ofrecieron ejemplos de su práctica profesional donde la CI era fundamental para sacar adelante una traducción y coincidieron en que no es posible el aprendizaje de una lengua sin aprender paralelamente su cultura. Esto demuestra que el nivel de CI del traductor incide verdaderamente en sus traducciones y por tanto sería necesario fomentar su adquisición temprana durante la etapa de formación.
- De ambas herramientas se desprende que el pensamiento generalizado sobre la relación entre la CI y las diferentes tipologías de traducción es que hay ciertos textos (técnicos y científicos) que conllevan menor carga cultural y por tanto el nivel de CI requerido es menor que en otros (literarios o jurídicos). No obstante, del conjunto de traductores entrevistados, los especializados en la rama técnica y médica sugirieron que la cultura se manifiesta en sus textos de manera más sutil y por tanto el conocimiento profundo de la otra cultura es vital para no pasarla por alto. Sería muy interesante profundizar más en este aspecto porque si bien estos traductores han arrojado un poco de luz sobre el tema, habría que entrevistar a una muestra más amplia para averiguar si este pensamiento es extensible a la rama técnica en general.

- A partir del punto anterior podemos concluir que los traductores encuestados albergaban una concepción bastante limitada de la CI que únicamente tiene en cuenta el aspecto textual de la misma dejando a un lado aspectos actitudinales y de interacción.

\section{APROXIMACIÓN AL CONCEPTO DE CI Y SU ADQUISICIÓN}

- Hemos recopilado una lista nada desdeñable de conocimientos, habilidades y actitudes que conformarían la CI proporcionada por los traductores encuestados que sin duda será de gran utilidad en nuestra propuesta de modelo CI para formación de traductores.

- La mayoría de traductores eligieron la etapa de formación universitaria como mejor momento para comenzar con un aprendizaje intercultural y seis de ocho entrevistados constataron que sería más apropiado comenzar este aprendizaje durante la infancia. Eran

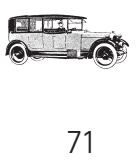

71 
conscientes de que la adquisición de la CI va estrechamente ligada a la idea de aprendizaje permanente y optaron por una metodología de enseñanza transversal donde el elemento cultural esté presente de forma homogénea en todas las asignaturas del currículum de TI combinado a su vez con materias específicas de cultura. Esta percepción de cómo debería ser la enseñanza de la CI enlaza con los métodos del EEES y creemos que habría que apuntar en esta dirección a la hora de diseñar los planes de estudio y programas didácticos del grado de TI.

A modo de valoración global, aunque somos conscientes de que las conclusiones recogidas a partir de los resultados de la muestra no son extensibles al resto de la población de traductores profesionales, sí que podríamos hablar de ciertas tendencias que invitan a continuar investigando para ampliar esta línea de estudio. El hecho de que la mayoría de traductores encuestados cuenten con un título universitario en TI contrasta con otros países donde la formación académica no está tan extendida como se desprende de los trabajos de Katan (2009b) y Pym et al. (2012). Por lo tanto, la formación de traductores en España es un campo donde se hace patente la necesidad de seguir investigando. Por otra parte, si los traductores sitúan la CI como una de las competencias más relevantes para el desarrollo de su profesión, creemos que haría falta un esfuerzo por que estuviera más presente tanto en los modelos de CT como en los propios planes de estudio de TI. Para lograr esto haría falta un impulso en la investigación sobre la CI en formación de traductores en España y esta investigación debería tener muy en cuenta la realidad del mercado de la traducción actual para minimizar en lo posible la brecha entre formación y profesión. Asimismo, cabría mencionar la necesidad de una formación intercul- tural del profesorado de TI y que esta formación estuviese incluida en el currículum universitario. Este aspecto lo aborda Paige (r986) que en los años 80 ya advertía sobre la necesidad de que la educación intercultural comenzaba por la formación de formadores y el desarrollo de la CI de los docentes para que éstos guiasen de forma adecuada a los estudiantes en este sentido:

The complexities and demands of culture learning require exceptional competencies of the trainer. These include a high degree of self-awareness and recognition of one's skills limitations, sensitivity of the needs of the learners, the ability to respond to the problems that culture learners encounter, and awareness of the ethical issues involved in cross-cultural training, conceptual/theoretical understanding, program-design skills, and research/evaluation skills. (Paige y Martin, I983 en Paige, I986: I35).

En lo que respecta al presente estudio, hemos cubierto un amplio espectro de cuestiones sobre la incidencia de la cultura en el trabajo diario del traductor profesional atendiendo a la variedad de especialidades de traducción que existen en el mercado. Si bien existen numerosos trabajos sobre la influencia del factor cultural en el proceso de traducción (Carbonell, I996; Leppihalme, I999; Godayol, 2003; Schäffner, 2003; Katan, 2004, 2009a; Bassnett, 2006; Witte, 2008), la mayoría se sitúa en un nivel teórico de investigación. No hemos encontrado estudios de corte empírico que hayan acudido directamente al traductor experto para comprobar, de primera mano, y con ejemplos reales de su práctica profesional, el modo en que la cultura se manifiesta en su rutina diaria de trabajo y el papel que desempeñaría la CI a este respecto. Por lo tanto, hemos intentado esbozar el perfil del traductor en este sentido, aunque somos conscientes de que ese retrato está aún incompleto. Si bien nos ayudará enormemente en el diseño de un mode- 
lo de CI aplicable a la formación de traductores, sería muy interesante continuar con esta línea de investigación tratando de ampliar la muestra de cuestionarios y el número de entrevistas para poder completar así este perfil del profesional relativo a la $\mathrm{CI}$ en su trabajo.

RECIBIDO EN OCTUBRE DE 2OI4 ACEPTADO EN SEPTIEMBRE DE 2015 VERSIÓN FINAL DE OCTUBRE DE 2015

\section{REFERENCIAS}

Bassnett, S. (2006). "Cultural, Colonialism and Gender Oriented Approaches to Translation». En: Keith Brown (ed.) Elsevier Encyclopaedia of Language E' Linguistics ( $2^{\mathrm{a}}$ ed.). Elsevier: Oxford, pp. 3II-3I4.

Byram, M. (1997). Teaching and Assessing Intercultural Communicative Competence. Clevedon: Multilingual Matters.

- (20I3). «Teaching communicative and intercultural competence». Lend. Lingua e nuova didattica, XLII/ 2, pp. 34-43.

Calvo, E.; N. Jiménez; I. Mendoza; M. Morón; N. Ponce y M. Enríquez (20I2). «El trabajo colaborativo en la clase de traducción: Un caso práctico». Revista UPO Innova, I, pp. 86-Io6. Disponible en: <http://www.upo.es/revistas/index.php/ upoinnova/article/view/88> [Consulta: $27 \mathrm{de}$ octubre de 20I4.]

Carbonell, O. (I996). «Lingüística, traducción y cultura». TRANS. Revista de Traductología, I, pp. I43-I5O.

Cea D’Ancona, M. A. (1996). Metodología cuantitativa: estrategias y técnicas de investigación social. Síntesis: Madrid.

Deardorff, D.K. (2006). «Identification and Assessment of Intercultural Competence as a Student Outcome of Internationalization». Journal of Studies in International Education, 10/3, pp. $24 \mathrm{I}-266$.

- (ed.). (2009). The Sage Handbook of Intercultural Competence. Sage: EE.UU.

European Union Lifelong Learning Programme (20I3). PICT: Promoting Intercultural Competence in Translators. Disponible en: <http://pict.icclanguages.eu/> [Consulta: 27 de octubre de 20I4.] García Izquierdo, I. (2oII) Competencia textual para la traducción. Valencia: Tirant Humanidades.

Gregorio, A. (2012). "Becoming a translator: the development of cultural and intercultural competence in Spain». Cultus, 5, pp.r54-I7I.

Gregorio y Gutiérrez (en prensa). «La competencia intercultural en Traducción: el perfil del estudiante en el contexto universitario de España».

Godayol, P. (2003). «El sujeto traductor multicultural: migrantes, subalternas, mestizas». En: Emilio Ortega Arjonilla (ed.) Panorama actual de la investigación en Traducción e Interpretación. Atrio: Granada, pp. I73-I8I.

Gutiérrez Bregón, S. (2009). La Competencia Intercultural en al aula de traducción e interpretación: Perspectiva del alumno. Trabajo fin de máster. Universidad de Granada: Granada.

Hueso, A. y M. J. Cascant (2012). Metodología y Técnicas Cuantitativas de Investigación. Cuadernos Docentes en Procesos de Desarrollo, I. Universidad Politécnica de Valencia: Valencia. Disponible en: <http://riunet.upv.es/bitstream/handle/10251/I7004/Metodologa\%20y\%2otcnicas\%20 cuantitativas\% $\%$ ode\% $\%_{2}$ oinvestigacin_6o6o. pdf ? sequence $=3>[$ Consulta: 27 de octubre de 20I4.]

Hurtado, A. (2007). «Competence-based Curriculum Design for Training Translators». The Interpreter and Translator Training, I/2, pp. I63-I95. Disponible en: <http://www.tandfonline.com/ toc/ritt2o/r/2\#.vE4g6vmG-So> [Consulta: 27 de octubre de 20I4.]

Katan, D. (2004). Translating Cultures. An Introduction for Translators, Interpreters and Mediators $\left(2^{\mathrm{a}}\right.$ ed.). St. Jerome: Mánchester.

- (2009a). «Translator Training and Intercultural Competence». En: S. Cavagnoli, E. Di Giovanni y R. Merlini (eds.) La ricerca nella comunicazione interlinguistica. Modelliteorici e metodologici, Franco Angeli: Milan, pp. 282-30.

- (2009b). "Translation theory and professional practice: a global survey of the great divide». Hermes, 42, pp. III-I54. Disponible en: <http:// download2.hermes.asb.dk/archive/download/ Hermes-42-7-katan_net.pdf> [Consulta: 24 de octubre de 20I4.] 
Kelly, D. (2002). «Un modelo de competencia traductora: bases para el diseño curricular». Puentes. Hacia nuevas investigaciones en la mediación intercultural, I, pp. 9-20. Disponible en: <http://www. ugr.es/ greti/puentes/puentesi/o2\%2oKelly.pdf> [Consulta: 24 de octubre de 20I4.]

- (2005). A Handbook for Translator Trainers. A Guide to Reflective Practice, St. Jerome: Manchester.

Latorre, A.; D. Del Rincón y J. Arnal (1996). Bases metodológicas de la investigación educativa. Grafiques 92: Barcelona.

Leppihalme, R. (1997). Culture Bumps: An empirical approach to the translation of allusions. Multilingual Matters LDT: Clevedon.

Oppenheim, A. N. (2000). Questionnaire design, interviewing and attitude measurement. ( $\left.8^{\mathrm{a}} \mathrm{ed}.\right)$. Continuum: Londres y Nueva York.

PACTE (2008). «First results of a Translation Competence Experiment: 'Knowledge of translation' and 'Efficacy of the translation process'». En: Kearns J. (ed.) Translator and interpreter Training: Issues, Methods and Debates, Continuum: Leeds, pp. I04-I27.

Paige, M. (I986). «Trainer Competencies: The Missing Conceptual Link in Orientation». International Journal of Intercultural Relations, Io, pp. I35-I58.

Pym, A.; F. Grin; C. Sfreddo y A. Chan (2012). Studies on translation and multilingualism: The status of the translation profession in the European Union. Comisión Europea. Disponible en: <http://

ec.europa.eu/dgs/translation/publications/studies/translation_profession_en.pdf> [Consulta: 24 de octubre de 20I4.]

Robinson, D. (2003). Becoming a translator. An introduction to the theory and practice of translation. $\left(2^{\mathrm{a}}\right.$ ed.) Routledge: Londres.

Ruiz-Olabuénaga, J. I. (20I2). Metodología de la investigación cualitativa. ( 5 a ed.). Universidad de Deusto: Bilbao.

Sandín Esteban, M. P. (2003). Investigación Cualitativa en Educación. Fundamentos y Tradiciones. McGraw-Hill/Interamericana de España: Madrid.

Schäffner, C. (2003). «Translation and Intercultural Communication: Similarities and Differences». Studies in Communication Sciences, 3/2, pp. 79-107.

Soriano, I. (2007). Evaluación de un programa de movilidad en la formación de traductores: expectativas, experiencias y grado de satisfacción de los participantes, profesores y gestores del intercambio $M G L U-U G R-U L P G C$. Tesis doctoral. Universidad de Granada: Granada.

Taylor, S.J y R. Bogdan (1987). Introducción a los métodos cualitativos de investigación: la búsqueda de significados. Paidós: Barcelona.

Witte, H. (2008). Traducción y percepción intercultural. Editorial Comares: Granada.

Yarosh,M.y L. Muies (20I2). «Developing translator's intercultural competence: a cognitive approach». Redit, 6, pp. 38-56. Disponible en: <http://www. redit.uma.es/Archiv/n6/3.pdf> [Consulta: 27 de octubre de 20I4.] 